\title{
Survey on the diagnosis and treatment of Clostridium difficile infection
}

\author{
Stofkova Z, Novakova E, Sadlonova V \\ Institute of Microbiology and Immunology, Comenius University in Bratislava, Jessenius Faculty \\ of Medicine in Martin, Slovakia. elena.novakova@uniba.sk
}

\begin{abstract}
BACKGROUND: The toxigenic strains of Clostridioides (Clostridium) difficile is the most common pathogen of nosocomial and antibiotic-related diarrhoea in healthcare facilities. Lately, there has been an increase in the incidence of $C$. difficile infection (CDI) cases in Slovakia.

MATERIALS AND METHODS: Retrospective analysis of the CDI appearance was carried out in the Zilina region. Additionally, an electronic survey focused on the diagnosis and treatment management of $C$. difficile infection was conducted among leading clinicians of the wards where CDI was present.

RESULTS: Eighty percent of clinicians reported that they were following the recommendations for diagnosis and treatment of $\mathrm{C}$. difficile infection in their everyday practice. The majority of leading physicians were from internal medicine wards $(50 \%)$. Most respondents stated that the laboratory results correlated with the clinical symptoms of patients. The first-choice treatment of $C$. difficile infections was reported to be oral vancomycin (in $21.7 \%$ ) and oral metronidazole (in $47.8 \%$ ). The estimate of first-choice treatment success rate was $80 \%$, while the recurrence rate and severe course was observed in $20 \%$. Vancomycin was the standard treatment for recurrent infection. It was administered orally either alone (31\%) or combined with metronidazole (38\%) or fidaxomicin (31\%). CONCLUSION: The results of the survey showed that recommendations for the diagnosis and treatment were implemented in the wards of hospitals and showed the awareness of the necessity of rapid diagnosis and early treatment of C. difficile infection in patients (Fig. 4, Ref. 30). Text in PDF www.elis.sk KEYWORDS: C. difficile, laboratory methods, recommendations for diagnosis and treatment of colitis caused by $C$. difficile, survey.
\end{abstract}

\section{Introduction}

The toxigenic strains of $C$. difficile are among the most common inducers of diarrhoea acquired in the hospital environment, and are considered to be the cause of a large proportion of antibiotic-related nosocomial diarrhoea which is a significant medical and economic burden in hospitals and other healthcare facilities. Over the last two decades, there has been an increase in the incidence of severe $C$. difficile infection (CDI) cases. New highly virulent $C$. difficile strains (e.g. ribotype 027) and other hypervirulent strains emerged (1). Several outbreaks have been caused by the North American Pulsed Field type 1 and polymerase chain reaction (PCR) ribotype 027 (NAP1/027) strain. This virulent strain has been associated with an increase in the production of toxins $\mathrm{A}$ and $\mathrm{B}$, fluoroquinolone resistance, and production of a binary toxin. The role of the binary toxin is not clear, but it may synergistically increase the virulence of toxins $\mathrm{A}$ and $\mathrm{B}$.

Institute of Microbiology and Immunology, Comenius University in Bratislava, Jessenius Faculty of Medicine in Martin, Malá Hora 11149/4B, SK-036 01 Martin, Slovakia

Address for correspondence: Z. Stofkova MD, PhD, Institute of Microbiology and Immunology, Comenius University in Bratislava, Jessenius Faculty of Medicine in Martin, Malá Hora 11149/4B, SK-036 01 Martin, Slovakia.

Phone: +421.904517772
From 2010 to 2017, the incidence in Slovakia increased from 0.9 to $20.6 / 10,000$ hospitalised patients (2). Hand hygiene is also proven to be effective in reducing the incidence of nosocomial infections (3).

An accurate and fast diagnosis of CDI is essential for optimal patient care and in preventing the spread of infection. It has been shown that rapid diagnosis has a positive impact on patients' care by reducing delays in initiating the isolation and treatment in confirmed CDI cases (4).

The European study (EUCLID, 2014) has shown suboptimal diagnosis of CDI caused by variations in testing strategies, as well as by the lack of clinical suspicion of the disease, and in turn by the lack of surveillance. Since then, hospitals have implemented optimal diagnostic algorithms, they increased and encouraged further implementation of optimal diagnostic methods for early and reliable diagnosis of $C$. difficile infections in order to avoid the spread of the disease (1).

A combination of high-sensitivity and higher-specificity methods is already used in the majority of diagnostic laboratories today (5).

The diagnosis of CDI is based on a combination of symptoms confirmed by microbiological evidence of toxins produced by $C$. difficile or toxigenic strains of C. difficile in faeces (6), without reasonable evidence for other cause of diarrhoea. Diarrhoea is a condition of having loose stools (corresponding to stool types 5-7 
in Bristol stool chart) in frequency of three stools in 24 or fewer consecutive hours (7). Risk factors for severe CDI are age over 65 years, current treatment with broad-spectrum antibiotics, and long-term hospitalization (longer than 3 weeks) (8).

Metronidalozole and vancomycin have been the mainstays of CDI treatment in recent years. Fidaxomicin is a macrocyclic antibiotic approved for the treatment of CDI in adults. Fidaxomicin is also associated with greater preservation of the intestinal microbiota as compared with vancomycin (9). Optimal treatment depends on the severity of the disease and the risk of recurrence (10). Treatment response is present when after therapy, either the stool frequency decreases or stool consistency improves, as well as when other parameters of disease severity (clinical, laboratory, radiological) improve and no new signs of severe disease develop (7).

A big problem is the recurrence of CDI which usually manifests as diarrhoea and other signs and symptoms of CDI, and is confirmed by recurrence or persistence of a positive stool test result for C. difficile or its toxins. Most recurrences, up to $25 \%$, occur within the first 30 days of completing a course of antiCDI antibiotic therapy, and may arise from a resumption of the primary infection (relapse) or a new exposure to C. difficile (reinfection) $(6,7)$.

Faecal microbiota transplant (FMT) is effective in the recurrent course of the disease (11).

FMT was integrated into European guidelines as a standard for the treatment of patients with multiple CDI relapses (7). The antibiotic stewardship in healthcare settings is needed (16)

\section{Methods}

The data were taken from the epidemiological information system (EPIS) of Slovakia. Reported CDI cases with colitis caused by $C$. difficile in population of Zilina self-governing region were processed from years 2015-2019. The involved districts of the Zilina self-governing region were Žilina, Bytča, Čadca, Kysucké Nové Mesto, Dolný Kubín, Trstená, Námestovo, Ružomberok, Liptovský Mikuláš, Martin, and Turčianske Teplice.

We have also conducted an electronic survey among leading clinicians from different wards where CDI was common. Electronic survey was focused on the diagnosis and treatment of $C$. difficile infection. The survey was carried out from March to June 2019 via electronic questionnaires which were sent to the leading clinicians of wards in Slovakia. The answers were filled in by 24/100 leading clinicians from various wards namely internal medicine (50\%), long-term care $(17 \%)$, intensive care $(17 \%)$, paediatrics and infectology ( $8 \%$ ), and oncology ( $8 \%$ ) from primary, secondary and tertiary hospitals. The questionnaires consisted of several questions concerning laboratory diagnosis and treatment of CDI, the use of current recommendations for the diagnosis and treatment of colitis caused by $C$. difficile $(8,10)$, testing methods, testing frequency in patients, testing of toxins, correlation of symptoms of CDI with laboratory results, treatment management according to the severity of the disease, treatment of CDI recurrences, and possibilities of other treatment methods (12).

\section{Results of appearance of CDI in Žilina region}

According to data reported to EPIS in Žilina region, the incidence of reported CDI increased. The incidence rate in 2015 was 4 patients per 10,000 inhabitants, it doubled in 2017, and increased up to 10 per 10,000 inhabitants in 2018, while in 2019, this number remained approximately on the same level with 10.2 CDI patients per 10,000 inhabitants. About $84 \%$ of CDI patients were hospital-associated infections (HAI-CDI), and $16 \%$ of CDI patients were community-associated cases (CA-CDI).

As displayed in Figure 1, the HAI-CDI cases per 100,000 inhabitants of Žilina region in 2019 were reported from internal wards, long-term care, intensive care units, surgery, pneumology, infectology, neurology and other wards (urology and oncology, etc.).

In the period from 2015 to 2019, the numbers of CDI cases per 100,000 inhabitants increased in Žilina region and Čadca district. In Martin district, the total number of reported CDI cases in population remained at the same level, while in 2019 there was even a slight decrease in the incidence of CDI cases. In 2019, $75.5 \%$ of CDI cases in population of Žilina district were older than 65 years, $12.5 \%$ were in the age group of 55-64 years, $8.5 \%$ were in the age group of 45-54 years, while $3.5 \%$ patients with CDI were reported among patients under 44 years. The proportion of females with CDI in 2019 was $55 \%$ while the proportion of males reached $45 \%$.

\section{Results of a survey of the diagnostics and treatment of CDI}

The survey questions were focused on the diagnosis and treatment of patients with colitis caused by toxigenic C. difficile.

Most of the clinicians who participated in the survey (80\%) stated that they were following the current recommendations for diagnosis and treatment of colitis caused by Clostridium difficile in their wards. The question was aimed at establishing which diagnostic testing methods the participants clinicians requested when CDI was suspected in their patients.

As to the laboratory testing of biologic samples, the glutamate dehydrogenase (GDH) assay and toxins A/B (EIA) were requested most frequently. In suspection of CDI in patients, a majority of

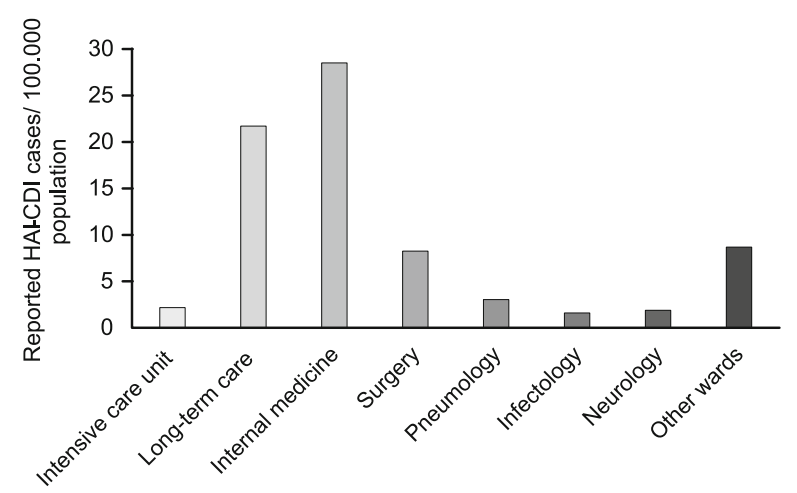

Fig. 1. Reported HAI-CDI cases in Zilina region in 2019. 


\section{0-846}

clinicians indicated GDH test along with A/B toxins. The realtime PCR (polymerase chain reaction) was not requested in the diagnosis of CDI. As to the testing the $C$. difficile toxin, the clinicians indicated the toxins A/B EIA test (immunochromatography) in $58.3 \%$, or enzyme-linked immunosorbent assay (ELISA) in $33.3 \%$ of patients' samples. Multi-step diagnostic algorithms are integrated in the laboratory practice.

The majority of clinicians ( $83.3 \%$ ) stated they requested diagnostic testing for CDI at the beginning and at the end of the CDI treatment, and $16.4 \%$ participants ordered the testing of biologic samples at the beginning, during, and after the treatment of CDI.

The turn-around time of laboratory testing methods was satisfying from the point of view of clinicians. The majority of clinicians reported that the results of laboratory tests for the evidence of toxins correlated well with patients' clinical symptoms of colitis caused by Clostridium difficile. A proportion of $50 \%$ of respondents stated that results of tests for the detection of $\mathrm{A} / \mathrm{B}$ toxins in patients correlated with the clinical signs of CDI in $80-90 \%$, while $33 \%$ of clinicians considered them to correlate in $60-80 \%$.

Eighty percent of participants in the survey presented that they have already been following current recommendations for therapy of colitis caused by $C$. difficile. The first-line therapy success rate estimated was $80 \%$ in clinical cure, $2-5 \%$ in persisting colitis, $1 \%$ in sepsis or megacolon, and $15 \%$ in recurrence. According to the participating clinicians, the success rate of first-line treatment in patients with CDI was $80 \%$. The recurrence of $C$. difficile infection occurred in $15 \%$ of CDI patients. The surveyed clinicians noted that recurrences were mostly common in their patients within 1 month or 2 months after completing the therapy of CDI ( $80 \%$ or $20 \%$, respectively).

The first-line therapy of $C$. difficile infections at a particular ward was reported to be oral vancomycin $(21.7 \%)$, oral metronidazole $(47.8 \%)$, or concomitant oral vancomycin with i.v. metronidazole $(17.4 \%)$.

Participants of the survey specified that in patients with CDI recurrence without SIRS or sepsis, they used vancomycin p.o. in 10 cases $(42 \%)$ as first-choice treatment, vancomycin p.o. concomitantly with metronidazole i.v. in $42 \%$, or fidaxomicin in $8 \%$. Vancomycin p.o. with descending pulse dosing was noted to be administered in $8 \%$ of the patients.

In cases of CDI recurrence with SIRS or sepsis, the clinicians reported to use vancomycin in (30 \%) as well as vancomycin p.o. and concomitant treatment with metronidazole i.v. in $40 \%$. They also reported treatment with fidaxomicin in $30 \%$.

As to the individual approach in CDI treatment, the clinicians mentioned diverse treatment strategies depending on whether there was a risk of severe course (e.g. severe leucocytosis), fulminant course (sepsis, toxic megacolon), if immunocompromised patients were involved, or continuance of antimicrobial treatment was necessary. Participating clinicians also noted that they used individual approach in treatment of colitis caused by $C$. difficile in patients with inflammatory bowel disease, i.e. those particularly susceptible to intestinal infections, also in older patients and those at high risk of developing enterococcal resistance (Fig. 2).

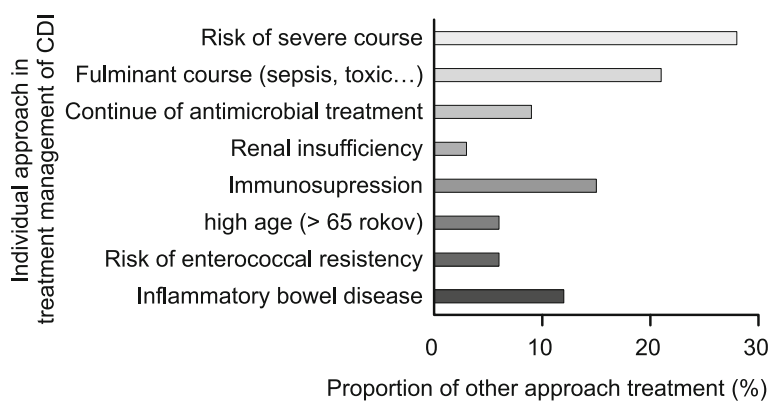

Fig. 2. Individual approach in the treatment management of colitis caused by $C$. difficile.

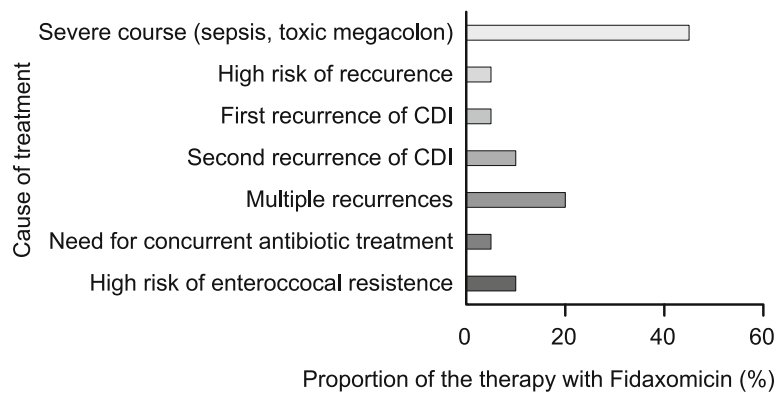

Fig. 3. Fidaxomicin used by clinicians.

The clinicians had already been using fidaxomicin in the treatment of patients with a severe course of CDI or in patients with recurrent $\mathrm{CDI}$ in cases that the intestinal microbiota had not been yet deteriorated (Fig. 3).

Almost $50 \%$ of clinicians had already been indicating the treatment with fidaxomicin p.o., especially in patients at high risk of severe disease, when the recurrent CDI could be risky, in case of severe course of infection (9 patients), in cases of first, second or multiple recurrences $(1,1$, or 4 patients, respectively), in case of high risk of recurrence ( 1 patient), in case of the need for continued antibiotic therapy ( 4 patients) and in patients at high risk of developing enterococcal resistance (2).

The clinicians noted that faecal microbiota transplant (FMT) was performed at their ward (25\%). The clinicians reported that faecal transplantation was carried out in patients with recurrent CDI (more than 2 or 3 recurrences).

\section{Discussion}

The incidence of CDI has also risen in Europe and all over the world. In a survey of 97 hospitals across 34 European countries, the incidence of $C$ difficile in hospitalized patients was 41 per 100,000 patient-days. In Canada's region of Quebec, the incidence in 2003 reached 92.2 cases per 100,000 population. CDI is more common in elderly people; old age may promote susceptibility to colonization and disease (13) According to Point Prevalence Survey (PPS) of healthcare-associated infections (HAI) carried out in the Slovak Republic in 2017, the most commonly detected microorganism was C. difficile (17.4\%) in comparison to the Eu- 


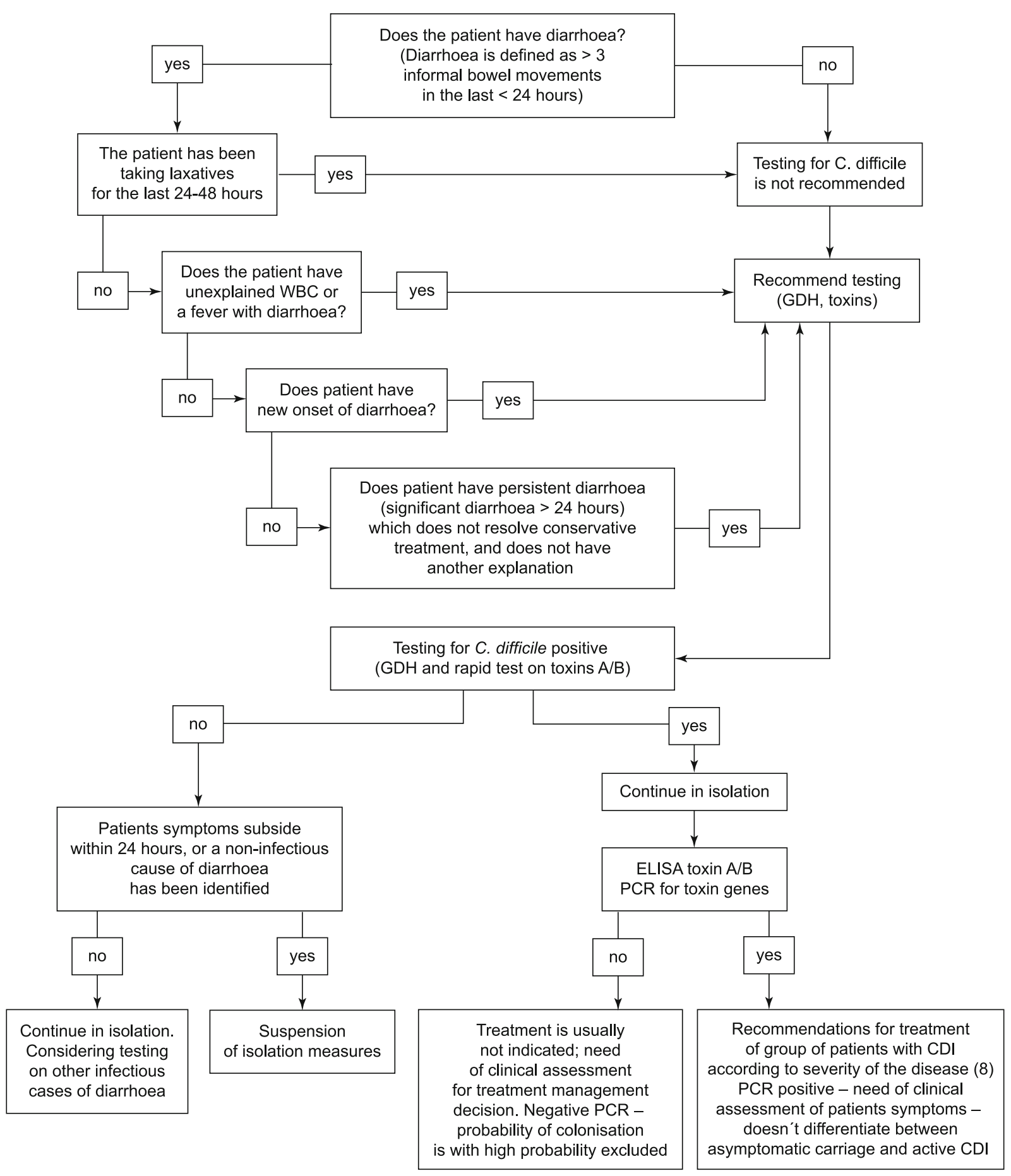

Fig. 4. Suggested diagnostic procedure for suspected CDI. Source: own processing according $(17,18)$.

ropean mean which was $7.3 \%$ Since 2012 , the incidence of the given pathogen has increased (from $1.7 \%$ to $17.4 \%$ ) due to its potential to survive and spread in the hospital environment (14). The incidence of clostridial infections in PPS in Slovakia (14.1\% of all nosocomial infections; $84.8 \%$ of gastrointestinal tract infections) was found to be high as compared to the European mean $(4.8 \%$ of all nosocomial infections; $44.6 \%$ of gastrointestinal infections) (15). The study revealed a decrease in frequency of CDI testing in comparison to the European mean. The median number of stool analyses for CDI in the Slovakia is 19 per 10,000 patient-days (14).

According to the study carried out by Novakova et al, $87.5 \%$ of participating hospitals used the recommended multi-step testing 
algorithm. The mean testing frequency in Slovakia was determined to be lower than that in the European Union (45.3 vs 36.5 tests per 10,000 patient-days (16)

In $2017,88.5 \%$ (1,715 cases) of hospital-associated cases of CDI in the Slovakia were reported from internal wards, $7.4 \%$ (144 cases) of HAI-CDI were from surgical wards, and 2.8 \% (54 cases) were from ICU (intensive care units) (2).

Several tests or test algorithms are used to improve the rapid and accurate diagnosis of CDI. There are three main drivers of CDI rates that can distort true incidence: diarrhoea sampling frequency, rate of CDI testing (testing/requesting frequency) and laboratory methods. The targeted testing of risk patients has also an impact on CDI rates (11).

No single commercial test can be used as a stand-alone test for diagnosing CDI as a result of inadequate positive predictive value at low CDI prevalence. Therefore, the use of two-step algorithm should be implemented in laboratories performing the CDI diagnosis. Samples without free toxin detected by toxins A and B EIA but with positive glutamate dehydrogenase EIA, the nucleic acid amplification test or toxigenic culture results need clinical evaluation to discern CDI patients from the asymptomatic carriers (7) In CDI diagnosis, the use of two- or three-step algorithms is recommended by ESCMID (European Society of Clinical Microbiology and Infectious Diseases) (6).

An electronic clinical support system has been implemented in some American hospitals to improve evidence-based testing and CDI diagnosis, which generated alerts related to the requirement for testing symptoms (more than 3 incidences of diarrhoea in 24 hours, leucocytosis, abdominal pain, and ileus), and alerts the system user of the need for CDI testing. Subsequent monitoring of the implementation of the decision-making system led to a reduction in inadequate testing of samples for CDI, cost of treatment and unnecessary antimicrobial therapy (17). The proposal of diagnostic procedure by patients suspected to have CDI (practical algorithm) was designed according to existing algorithms $(17,18)$. The algorithm would not replace the judgment of clinicians (Fig. 4).

In GDH-positive and A/B toxin -negative stool samples, the PCR tests for the detection of toxin genes by nucleic acid amplification test or by toxigenic culture are recommended (7) For patients who are. C. difficile-positive by molecular-biological assay (PCR) but who are toxin-negative by immunoenzymatic methods, may be referred to as "C. difficile" carriers (20), as PCR determines the genes for toxin production (21). The reference methods (toxigenic culture and cytotoxin neutralisation assay) are not standard in routine laboratory practice, as they require special equipment, staff experience, and time, especially for toxicity testing.

Another issue is the number of samples per patient that should be submitted for testing. The diagnostic gain of repeated testing within a 7-day period with both toxin A/B EIA and PCR was demonstrated to be very low. However, in cases of ongoing clinical suspicion during an endemic situation, the submission of a repeated testing may be justified (6) In suspicion of CDI in patients, an anaerobic culture is established simultaneously for the presence of $C$. difficile in the tested sample of the patient. Formed stool samples should not be tested for CDI as these do not meet the clinical criteria of CDI, (only unformed stool samples should be tested). However, sometimes only solid parts of diarrhoeal faeces may be collected and submitted for $C$. difficile testing. An exception has to be made for patients suspected of CDI who have ileus. In these patients, a rectal swab can be used with adequate sensitivity and specificity for (toxigenic) culture, real-time PCR or GDH (7).

Metronidazole is used as the drug of the first choice in patients with mild CDI. This finding was stated also in the survey. First-line therapy of $C$. difficile infections at a particular ward was reported to be oral vancomycin, oral metronidazole, or oral vancomycin with i.v. metronidazole, namely in $21.7 \%, 47.8 \%$ or $17.4 \%$ of CDI cases, respectively. The estimate of first-line therapy success rate was $85 \%$ in clinical cure, $2-5 \%$ in persisting colitis, $1 \%$ in sepsis or megacolon, $5-10 \%$ in recurrence, and $3 \%$ resulted in death. In patients with a moderate to severe episode of CDI, vancomycin is used as the first-line drug and fidaxomicin is used as the second-line drug. In the case of a severe episode of CDI, vancomycin is used as the drug of choice. Fidaxomicin is a secondline drug in cases of a severe episode of CDI (8).

Clinicians noted an individual approach in the treatment of CDI in patients at risk of severe or fulminant course of infection, in case of immunosuppressed patients, as well as in patients with inflammatory bowel disease.

A study by Nylund et al suggested an increase in CDI among hospitalized patients. With conditions such as IBD (inflammatory bowel disease) and immunosuppression, at risk are individual hospitalized patients that require antibiotic administration (22). Patients with chronic inflammatory bowel disease (IBD) have a significantly increased risk of clinically relevant clostridial infection (CDI). In turn, CDI can increase IBD activity. IBD patients with a proven CDI should be treated primarily with vancomycin (23).

Vancomycin and fidaxomicin are minimally absorbed from the intestinal tract and have high achievable concentrations in the intestinal lumen. Meanwhile, fidaxomicin has the ability to maintain the physiological microbiome, and the prevention of recurrences is higher in comparison to vancomycin (24).

Standard treatments for recurrent infection were vancomycin administered orally either alone (31\%) or combined with metronidazole $(38.5 \%)$ or fidaxomicin $(31 \%)$. Fidaxomicin has been used almost once in $50 \%$ of treatments in the survey. Recent studies showed also that most recurrences occur within the first 6 weeks after finishing the antibiotic treatment of CDI (25). Approximately $20-27 \%$ of patients treated for the first episode of $C$ difficile colitis relapse after successfully completing the therapy, typically 3 days to 3 weeks after treatment was ended. Patients who relapse once are at an even greater risk for further relapses; the relapse rate for patients with 2 or more relapses is $65 \%$ (26). Fidaxomicin and vancomycin reach high concentrations in faeces. Fidaxomicin has a lower recurrence rate of CDI in comparison to vancomycin (27). The number of patients with recurrent CDI is still growing and currently used therapeutic approaches are not always effective (28). In a relapsing CDI, faecal microbiome transfer is an effective therapeutic measure.

A proportion of respondents $(25 \%)$ have used faecal microbiota transplant (FMT) in selected cases at their ward according to 
the survey. The faecal microbiota transplant is a novel treatment in CDI with multiple recurrences (29)- Most recurrences, up to $25 \%$, occur within the first 30 days of completing the course of anti-CDI antibiotic therapy, and may arise from a resumption of the primary infection (relapse) or from a new exposure to C. difficile (re-infection) $(6,7)$.

The management of recurrent clostridial infections is very demanding and expensive, it impacts mostly elderly polymorbid patients (30). It is necessary to provide patients with timely appropriate antibiotic therapy while special attention should be paid to early selection of those who are at high risk of the disease in terms of rapid selection of the most appropriate therapy (8). In the context of CDI, there is a need for continuous awareness of the issue, as well as optimization and evaluation of diagnostic procedures and algorithms and an early onset of the treatment that can help reduce the incidence, morbidity and mortality of $C$. difficile infection (6).

\section{Conclusion}

The aim of the survey was to analyse the use of recommendations for the diagnosis and treatment of CDI among clinicians from different wards. The majority of respondents $(80 \%)$ declared that they were performing tests according to the recommendations for the diagnosis and treatment of colitis caused by Clostridium difficile. Most of the clinicians stated that the testing results of CDI correlated in most cases with the clinical symptoms of the patients with CDI. They reported using algorithms for the diagnosis (toxin testing together with GDH screening) and treatment (80\%).

The first-line therapy of $C$. difficile infections at a particular ward was reported to be oral vancomycin $(21.7 \%)$, oral metronidazole $(47.8 \%)$ or oral vancomycin with i.v. metronidazole (17.4\%).

The first-line therapy success rate estimate was $80 \%$, while in $15 \%$ of patients, the clinicians estimated recurrence. Vancomycin administered orally was the standard treatment for recurrent infection, either alone $(31 \%)$ or combined with metronidazole $(38 \%)$ or fidaxomicin (31\%). Fidaxomicin has been used in treatment management strategy in $50 \%$. A proportion of clinicians $(25 \%)$ have used faecal microbiota transplant (FMT) in selected cases at their ward. The results of the survey showed that recommendations for diagnostic methods are in most cases implemented in the diagnosis and treatment of CDI in wards of hospitals. Our survey indicated that leading clinicians are highly aware of $C$. difficile infections, however reveals differences in diagnostic and therapeutic procedures.

\section{References}

1. Davies KA, Longshaw CM, Davis GL et al. Underdiagnosis of Clostridium difficile across Europe: the European, Multicentre, Prospective, Biannual, Point-Prevalence Study of Clostridium difficile Infection in Hospitalised Patients with Diarrhoea (EUCLID). Lancet Infect Dis 2014; 14 (12): 1208-1219.

2. Novak M, Mazakova I, Sadlonova V et al. Appearance of Clostridium difficile infections in health care institutions in Slovakia and in the district of Martin. Acta Med Martiniana 2019; 18 (2).
3. Novak M, Breznicky J, Kompanikova $\mathbf{J}$ et al. Impact of hand hygiene knowledge on the hand hygiene compliance. Med Glas (Zenica) 2020; 17 (1): 194-199.

4. Gateau C, Couturier J, Coia J, Barbut F. How to: diagnose infection caused by Clostridium difficile. Clin Microbiol Infect 2018; 24 (5): 463-468.

5. Murad YM, Perez J, Ybazeta G et al. False Negative Results in Clostridium difficile Testing. BMC Infectious Diseases volume 16, Article number: 430 (2016).

6. Debast MP, Bauer EJ, Kuijper SB. European Society of Clinical Microbiology and Infectious Diseases: update of the treatment guidance document for Clostridium difficile infection. Clin Microbiol Infect 2014; 20 (Suppl 2): 1-26.

7. Crobach MJT., Planche T, Eckert C et al. European Society of Clinical Microbiology and Infectious Diseases: update of the diagnostic guidance document for Clostridium difficile infection. Clin Microbiol Infect 2016; 22 (Suppl 4): S63-81.

8. Jarčuška Pet al. Odporúčaný postup diagnostiky a liečby kolitídy spôsobenej Clostridium difficile. Via pract 2015; Suppl 1 (12).

9. Freeman J, Vernon J, Pilling $\mathbf{S}$ et al. Five-year Pan-European, longitudinal surveillance of Clostridium difficile ribotype prevalence and antimicrobial resistence: the extended ClosER study. Eur J Clin Microbiol Infect Dis 2020; 39 (1): 169-177.

10. Beneš J, Husa P, Nyč O, Polívková S. Doporučený postup diagnostiky a léčby kolitidy vyvolané Clostridium difficile. Společnost infekčníhom lékařství 2014. https://www.infekce.cz/dpCDI14.

11. Davies K, Perelle S, Gilbert $M$ et al. Production of a complete binary toxin (actin-specific ADP-ribosyltransferase) by Clostridium difficile CD196. Infect Immun 1997; 65: 1402-1407.

12. Bruensing et al. Management of Clostridium difficile infections at German intensive care units - results from a survey among intensivists, $\mathrm{Z}$ Gastroenterol 2018; 56 (6): 551-560.

13. Bauer MP, Notermans DW, van Benthem BH et al. Clostridium difficile infection in Europe: a hospital-based survey. Lancet 2011; 377 (9759): 63-73.

14. Štefkovičová M, Litvová S, Mikas J et al. Druhé bodové prevalenčné sledovanie nozokomiálnych nákaz na Slovensku ako súčast’ európskeho sledovania [Second point prevalence survey of healthcare-associated infections in the Slovak Republic as a part of the european survey] Zdravotnícke Listy 2019; 7 (4).

15. Suetens C, Latour K, Kärki T et al. Prevalence of healthcare-associated infections, estimated incidence and composite antimicrobial resistance index in acute care hospitals and long term care facilities: results from two european point prevalence surveys, 2016 to 2017. Eurosurveillance, 2018; 23 (46): [online]. https://www.eurosurveillance.org/content/10.2807/1560-7917.ES.2018.23.46.1800516.

16. Novakova E, Stefkovicova M, Kopilec MG et al. The emergence of Clostridium difficile ribotypes 027 and 176 with a predominance of the Clostridium difficile ribotype 001 recognized in Slovakia following the European standardized Clostridium difficile infection surveillance of 2016. Int J Infect Dis 2020; 90: 111-115.

17. Aitken $\mathbf{S}$, Cantu $\mathbf{S}$, Cortes $\mathbf{J}$ et al. Assessment and Management of Clostridioides difficile Infections (CDI) - Adult. MD Anderson Cancer Center, Department of Clinical Effectiveness, University of Texas, 13 p. 2018. 


\section{0-846}

18. Miller B, O’Neal C, Hameret D. Using Clinical Decision Support to Improve Evidence Based Testing and Diagnosis of Clostridium difficile Infection. Open Forum Infect Dis 2017; 4 (Suppl 1): S396.

19. Nylund CM, Goudie A, Garza JM, Fairbrother G, Cohen MB. Clostridium difficile infection in hospitalized children in the United States. Arch Pediatr Adolesc Med 165 (5): 451-457.

20. Lübbert Ch, John E, von Müller L. Clostridium difficile infection -guideline-based diagnosis and treatment. Dtsch Arztebl Int 2014; 111 (43): 723-731.

21. Robotham J, Wilcox M. Updated Guidance on the Diagnosis and Reporting of Clostridium Difficile. NHS, Advisory Committee on Antimicrobial Resistance and Healthcare Associated Infection (ARHAI), 2012. Available: https:/www.gov.uk/government/uploads/system/uploads/attachment_data/file/146808/dh_133016.pdf.pdf.

22. Burnham CA, Carroll KC. Diagnosis of Clostridium difficile Infection: an Ongoing Conundrum for Clinicians and for Clinical Laboratories. Clin Microbiol Rev 2013; 26 (3): 604-630.

23. Stallmach A, Reuken PA, Teich N. Aktuelle Konzepte zur Diagnostik und Therapie der Clostridioides [Clostridium]-difficile-Infektion bei Patienten mit chronischentzündlichen Darmerkrankungen Advances in the diagnosis and treatment of Clostridioides [Clostridium] difficile infections in inflammatory bowel disease. Gastroenterologie 2018; 56, s. 1369-1377.
24. Maxwell-Scott HG, Goldenberg SD. Existing and investigational therapies for the treatment of Clostridium difficile infection: A focus on narrow spectrum, microbiota-sparing agents. Med Mal Infect 2018; 48 (1): 1-9

25. Kelly CP. Can we identify patients at high risk of recurrent Clostridium difficile infection? Clin Microbiol Infect 2012; 18 (Suppl 6): 21-27.

26. Johnson S. Recurrent Clostridium difficile infection: a review of risk factors, treatments, and outcomes. J Infect 2009; 58 (6): 403-410.

27. Beneš J, Polívková S. Antibiotic treatment of clostridial colitis. Epidemiol Microbiol Imunol 2016; 65 (1): 15-24.

28. Vejmelka J, Kohout $\mathbf{P}$, Koten $\mathbf{J}$ et al. Fekální bakterioterapie a nové cesty v léčbě klostridiové střevní infekce (Fecal microbiota transplantation and novel therapies for Clostridium difficile infection). Prakt Lek 2014; 94 (4): 195-200.

29. Šturdík I, Hlavaty T, Payer J. Fecal microbiota transplantation. Vnitr̆ Lék 2016; 62 (2): 147-151.

30. Sarvašová $\mathbf{M}$, Koščalová $\mathbf{A}$, Sabaka $\mathbf{P}$ et al. Fekálna mikrobiálna terapia skúsenosti na Slovensku. Ružgad - Ružinovský gastroenterologický deň 2019. http://ruzgad.sk/fekalna-mikrobialna-terapia-skusenosti-na-slovensku/.

Received April 30, 2020. Accepted May 15, 2020. 